\title{
Estimativa de temperaturas favoráveis para o desenvolvimento da avicultura
}

O presente estudo teve como objetivo ajustar os dados de temperaturas ocorridas no município de Santo Antônio de Leverger/MT, aplicando-se diversas Funções Densidade de Probabilidade. Foram analisadas as séries históricas de temperaturas mínimas e máximas diárias, obtidas no Banco de Dados Meteorológicos para o Ensino e Pesquisa (BDMEP) do Instituto Nacional de Meteorologia. Para a pesquisa considerou-se o período de janeiro de 1987 a dezembro de 2019, registradas na Estação Meteorológica Convencional Padre Ricardo Remetter. Com auxílio da planilha EXCEL e do programa R, obtiveram-se as principais estatísticas descritivas dos dados, baseadas nas medidas de posição e dispersão e elaboraram-se os diagramas de caixa para cada mês e tipo de temperatura extrema com a finalidade de constatar as características da distribuição. Em seguida, os dados mensais de cada temperatura foram agrupados em dez (10) classes, adotando-se a Regra de Sturges, para facilitar a definição do modelo probabilístico. Os dados de temperaturas mínimas e máximas foram ajustados às cinco distribuições probabilísticas, a seguir: Normal, Log Normal, Gama, Gumbel e Weibull. Na sequência, foi verificada a aderência dos dados à essas distribuições aplicando-se o teste de Qui-Quadrado. Foi constatado que os valores da temperatura mínima diária se ajustaram adequadamente à distribuição Normal para todos os meses, porém, os valores de temperatura máxima somente nos meses de janeiro até abril e de outubro até dezembro. Já, os meses de maio até setembro foram modelados aplicando-se a distribuição Gama. Logo, com base nas distribuições representativas dos meses para cada temperatura, elaborou-se um gráfico de barras empilhadas para estimar a probabilidade de ocorrência da temperatura mensal, classificada em muito fria, adequada e muito quente para a avicultura. Concluiu-se que As Funções Densidade de Probabilidade (FDP) foram adequadas, precisas e confiáveis para o ajuste dos dados diários de temperaturas extremas (mínima e máxima) em cada mês do ano, no município de Santo Antônio de Leverger-MT. Os modelos obtidos possibilitaram definir os limites favoráveis para o desenvolvimento da avicultura e estimar a probabilidade de ocorrência das três categorias de temperaturas nesse município. A metodologia adotada apresentou-se como excelente ferramenta para predizer os valores de temperaturas mínimas e máximas ao longo do ano no referido município, auxiliando no planejamento da construção de aviários e seu respectivo sistema de ventilação.

Palavras-chave: Ambiência; Bem-estar animal; Funções densidade de probabilidade.

\section{Estimate of favorable temperature for the development of poultry farming} \begin{abstract}
The present study aimed to adjust the temperature data that occurred in the municipality of Santo Antônio de Leverger/MT, applying several Probability Density Functions. The historical series of minimum and maximum daily temperatures, obtained from the Meteorological Database for Teaching and Research (BDMEP) of the National Institute of Meteorology, were analyzed.
For the research, the period from January 1987 to December 2019 was considered, registered at the Conventional Meteorological Station Padre Ricardo Remetter. With the aid of the EXCEL spreadsheet and the R program, the main descriptive statistics of the data were obtained, based on the position and dispersion measures and the box plot diagrams for each month and type of extreme temperature were elaborated in order to verify the characteristics of the distribution. Then, the monthly data for each temperature were grouped into ten (10) classes, adopting the Sturges Rule, to facilitate the definition of the probabilistic model. The minimum and maximum temperature data were adjusted to the five probabilistic distributions, as follows: Normal, Normal Log, Gamma, Gumbel, and Weibull. Subsequently, the data adherence to these distributions was verified by applying the Chi Square test. It was found that the values of the minimum daily temperature adjusted adequately to the Normal distribution for all months, however, the values of maximum temperature only in the months of January to April and from October to December. The months from May to September were modeled using the Gamma distribution. Therefore, based on the representative distributions of the months for each temperature, a December. stacked bar gro Probability Density Functions (FDP) were adequate, accurate and reliable for adjusting the daily data of extreme temperatures (minimum and maximum) in each month of the year, in the municipality of Santo Antônio de Leverger-MT. The obtained models made it possible to define favorable limits for the development of poultry and to estimate the probability of occurrence of the three temperature categories in this municipality. The adopted methodology was presented as an excellent tool to predict the values of minimum and maximum temperatures throughout the year in that municipality, assisting in the planning of the construction of aviaries and their respective ventilation system.
\end{abstract}

Keywords: Ambience; Animal welfare; Probability density functions.

Topic: Engenharia Agrícola

Reviewed anonymously in the process of blind peer.

Pedro Hurtado de Mendoza Borges (iD)

Universidade Federal de Mato Grosso, Brasil

http://lattes.cnpq.br/0310556104378957

http://orcid.org/0000-0001-7603-8775

pborges@ufmt.br

Zaíra Morais dos Santos Hurtado de Mendoza (iti)

Universidade Federal de Mato Grosso, Brasil

http://lattes.cnpq.br/7829408545924908

http://orcid.org/0000-0002-0930-7928

zaira@ufmt.br

Pedro Hurtado de Mendoza Morais

Universidade Federal de Mato Grosso, Brasil

http://lattes.cnpq.br/9517941254638374

http://orcid.org/0000-0003-2431-9262

pedromorais08@hotmail.com

DOI: 10.6008/CBPC2179-6858.2020.006.0010
Received: 07/10/2020

Approved: 19/11/2020
Referencing this:

BORGES, P. H. M.; MENDOZA, Z. M. S. H.; MORAIS, P. H. M.. Estimativa de temperaturas favoráveis para o desenvolvimento da avicultura. Revista Ibero Americana de Ciências Ambientais, v.11, n.6, p.112119, 2020. DOI: http://doi.org/10.6008/CBPC21796858.2020 .006 .0010 


\section{INTRODUÇÃO}

O ambiente externo influencia significativamente a temperatura interna dos aviários, principalmente quando não há um sistema de ventilação eficiente. A ocorrência de temperaturas diárias elevadas ou baixas, fora da faixa de conforto, pode trazer como consequência perdas produtivas e reprodutivas consideráveis na avicultura devido ao estresse térmico causado (SALGADO et al., 2006; NAVAS et al., 2016). Na tentativa de minimizar os efeitos negativos dessa variável, podem ser realizadas diversas modificações construtivas e ambientais nas instalações (VITORASSO et al., 2009; COELHO et al., 2015). Entretanto, para elevar o desempenho dessas modificações seria necessária uma adequada precisão na predição dos valores de temperatura. Devido a isso, estimar o comportamento probabilístico dessa variável é de suma importância para elaborar o projeto dos aviários e seu respectivo sistema de ventilação.

As Funções Densidade de Probabilidade com diferentes números de parâmetros têm sido aplicadas em diversos estudos relacionados às ciências agrárias tais como, climatologia, meteorologia, hidrologia e ambiência, entre outras (ASSIS et al., 2004; ASTOLPHO et al., 2005; SILVA et al., 2005; SANSIGOLO, 2008; CALDEIRA et al., 2015; JERSZURKI et al., 2015; BORGES et al., 2016). Esses trabalhos evidenciaram a elevada eficiência das referidas funções e sua utilidade como ferramenta potencial para estimar a frequência e a probabilidade de ocorrer eventos extremos de variáveis meteorológicas. Porém, os autores são unânimes ao ressaltar que para obter elevado desempenho e adequada precisão nos resultados, as Funções Densidade de Probabilidade devem ser desenvolvidas com base em longas séries históricas de dados confiáveis.

Os trabalhos consultados permitem inferir que as Funções Densidade de Probabilidade constituem uma ferramenta útil para a modelagem de dados meteorológicos e que o seu uso é promissor para predizer as temperaturas extremas mensais, baseando-se em informações climáticas de séries históricas, disponíveis nos bancos de dados do Instituto Nacional de Meteorologia (INMET). Logo, esta pesquisa fundamenta-se na hipótese de que esses modelos probabilísticos podem servir como excelente ferramenta de análise para fornecer os critérios necessários em relação ao ambiente térmico externo de aviários, auxiliando a tomada de decisões para elaborar o projeto do sistema de ventilação que minimize os efeitos do estresse animal. Essa problemática motivou a realização do presente estudo, que como objetivo modelar os dados de séries históricas de temperaturas mínimas e máximas ocorridas no município de Santo Antônio de Leverger-MT, aplicando-se diversas Funções Densidade de Probabilidade, que possibilitem definir, com adequada precisão e confiabilidade, os limites favoráveis para o desenvolvimento da avicultura em cada mês do ano.

\section{MATERIAIS E MÉTODOS}

O material utilizado neste estudo foram as séries históricas de temperaturas mínimas e máximas diárias, disponíveis no Banco de Dados Meteorológicos para o Ensino e Pesquisa (BDMEP) do Instituto Nacional de Meteorologia (INMET), no período de janeiro de 1987 a dezembro de 2019. Os dados foram registrados na Estação Meteorológica Convencional Padre Ricardo Remetter, código OMM 83364, operante

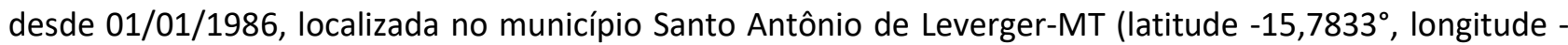


56,0667 ${ }^{\circ}$ altitude 140 m). O clima tropical da região foi classificado como Aw (PEEL et al., 2007). Na estação, a temperatura média anual e a pluviosidade média anual oscilam, respectivamente, em torno de $26,1^{\circ} \mathrm{C}$ e 1267 mm (INMET, 2020).

Os valores foram armazenados em planilhas eletrônicas, Microsoft EXCEL e em seguida, utilizou-se o módulo desenvolvedor VBA ('Visual Basic for Applications') para a elaboração de duas macros. A primeira macro possibilitou classificar os dados das séries históricas de temperaturas mínimas e máximas diárias, de acordo com o mês e dia de cada ano. A segunda macro auxiliou no resumo dos dados mensais das respectivas séries, de forma independente. Logo após, foram realizados os procedimentos gráficos e analíticos com auxílio do programa R (R CORE TEAM, 2020). Sequencialmente, calcularam-se as principais estatísticas descritivas, baseadas nas medidas de posição e dispersão. Posteriormente, com a finalidade de constatar a distribuição dos dados, elaboraram-se os diagramas de caixa para as temperaturas extremas, em função do mês. Por fim, visando facilitar a definição do modelo probabilístico, os dados mensais de cada temperatura foram agrupados em dez classes, conforme a Regra de Sturges.

Tomando como referência os estudos de Assis et al. (2004), Silva et al. (2005), Sansigolo (2008), Araújo et al. (2010), Salgado et al. (2010), Caldeira et al. (2015), Jerszurki et al. (2015) e Borges et al. (2016), os dados de temperaturas mínimas e máximas foram ajustados às cinco distribuições probabilísticas, a seguir: Normal, Log Normal, Gama, Gumbel e Weibull. Na sequência, foi verificada a aderência dos dados à essas distribuições aplicando-se o teste de Qui-Quadrado. Logo, com base nas distribuições representativas dos meses para cada temperatura, elaborou-se um gráfico de barras empilhadas para estimar a probabilidade de ocorrência da temperatura mensal. Para classificar as temperaturas, adotou-se a escala recomendada por Salgado et al. (2010), cuja descrição e limites são: muito baixa (inferior a $22{ }^{\circ} \mathrm{C}$ ), adequada (de 22 a $32{ }^{\circ} \mathrm{C}$ ) e muito alta (superior a $32^{\circ} \mathrm{C}$ ).

\section{RESULTADOS E DISCUSSÃO}

As principais estatísticas descritivas mensais para a temperatura mínima diária, registradas na estação meteorológica de Santo Antônio de Leverger-MT constam na Tabela 1. Na referida Tabela, observase que os valores das medidas de posição foram semelhantes para todos os meses. Nota-se, ainda, que nos meses de maio até setembro os valores médios da temperatura mínima foram inferiores ao limite mínimo de $22{ }^{\circ} \mathrm{C}$, sugerido para a avicultura. Também, nesse período as medidas de dispersão atingiram os maiores valores, indicando elevada flutuação da temperatura mínima, conforme mostram o desvio padrão maior do que $3^{\circ} \mathrm{C}$ e o coeficiente de variação acima de $9 \%$.

Tabela 1: Estatísticas descritivas mensais para a temperatura mínima diária na Estação Meteorológica Convencional Padre Ricardo Remetter, localizada no município de Santo Antônio de Leverger-MT.

\begin{tabular}{|l|l|l|l|l|l|l|}
\hline \multirow{2}{*}{ Meses } & \multicolumn{5}{l}{ Estatística descritiva } \\
\cline { 2 - 7 } & Média & Mediana & Desvio Padrão & Coeficiente de Variação (\%) & Erro Padrão & Intervalo de confiança (95\%) \\
\hline Janeiro & 23,4764 & 23,5000 & 1,1928 & 5,0810 & 0,0376 & $\pm 0,0737$ \\
\hline Fevereiro & 23,3834 & 23,4000 & 1,0955 & 4,6850 & 0,0360 & $\pm 0,0706$ \\
\hline Março & 23,3516 & 23,4000 & 1,3360 & 5,7212 & 0,0418 & $\pm 0,0819$ \\
\hline Abril & 22,6642 & 22,9000 & 1,8319 & 8,1433 & 0,0584 & $\pm 0,1144$ \\
\hline Maio & 19,5936 & 20,3000 & 3,0025 & 15,3239 & 0,0942 & $\pm 0,1845$ \\
\hline
\end{tabular}




\begin{tabular}{|l|l|l|l|l|l|l|}
\hline Junho & 17,4750 & 17,6000 & 2,6779 & 15,3241 & 0,0880 & $\pm 0,1724$ \\
\hline Julho & 15,8399 & 16,0000 & 3,2576 & 20,5658 & 0,1062 & $\pm 0,2081$ \\
\hline Augusto & 17,6434 & 17,6000 & 3,5150 & 19,9226 & 0,1139 & $\pm 0,2232$ \\
\hline Setembro & 20,8012 & 21,1000 & 3,2762 & 15,7501 & 0,1078 & $\pm 0,2114$ \\
\hline Outubro & 23,0083 & 23,1000 & 2,0781 & 9,0320 & 0,0660 & $\pm 0,1295$ \\
\hline Novembro & 23,2806 & 23,3000 & 1,7394 & 7,4714 & 0,0563 & $\pm 0,1103$ \\
\hline Dezembro & 23,5273 & 23,5000 & 1,3349 & 5,6739 & 0,0423 & $\pm 0,0829$ \\
\hline Total anual & 21,1975 & 22,5000 & 3,5655 & 16,8203 & 0,0330 & $\pm 0,0648$ \\
\hline
\end{tabular}

As medidas de posição anuais para a temperatura diária mínima do munícipio de Santo Antônio de Leverger-MT estimadas neste trabalho foram superiores às obtidas por Salgado et al. (2010) no município Mirante do Paranapanema-SP. Porém, as medidas de dispersão deste estudo foram inferiores, evidenciando maior precisão dos resultados nesta pesquisa. Costa et al. (2012) obtiveram médias de temperaturas mínimas diárias próximas a $23,7{ }^{\circ} \mathrm{C}$ para o município de Bananeiras-PB, valor superior ao limite mínimo. De acordo com os resultados deste estudo, deduz-se que as condições térmicas do município avaliado não foram favoráveis para a avicultura durante os meses de inverno e que uso de ventilação natural durante esse período requer especial atenção, pois as perdas produtivas e reprodutivas podem ser significativas.

Na Figura 1 apresentam-se os diagramas de caixa ('box plot') para a temperatura diária mínima, em função do mês. Nessa Figura foram adicionados os pontos correspondentes à média mensal, e a linha horizontal no valor $21,1975{ }^{\circ} \mathrm{C}$ para indicar o valor médio anual. Pode-se constatar pouca variabilidade dos valores de temperaturas durante os meses de janeiro até abril, outubro, novembro e dezembro, períodos com menor amplitude térmica, em que a maioria dos valores de temperatura situam-se entre 22 e $25{ }^{\circ} \mathrm{C}$. Assim, durante esses meses, os valores da temperatura mínima diária não devem interferir negativamente no desempenho produtivo e reprodutivo das aves. Porém, nos meses de maio, junho, julho e agosto, a situação de conforto animal por causa da temperatura mínima pode ser considerada crítica, pois houve elevada amplitude térmica e mais de $75 \%$ dos valores de temperatura foram inferiores a $22{ }^{\circ} \mathrm{C}$, limite mínimo recomendado para a avicultura. De forma geral pode-se verificar para todos os meses pouca diferença entre a média e a mediana, bem como entre os quartis, caracterizando uma distribuição simétrica dos dados, podendo diminuir a probabilidade de ocorrer temperaturas mínimas diárias muito diferentes de um dia para outro.

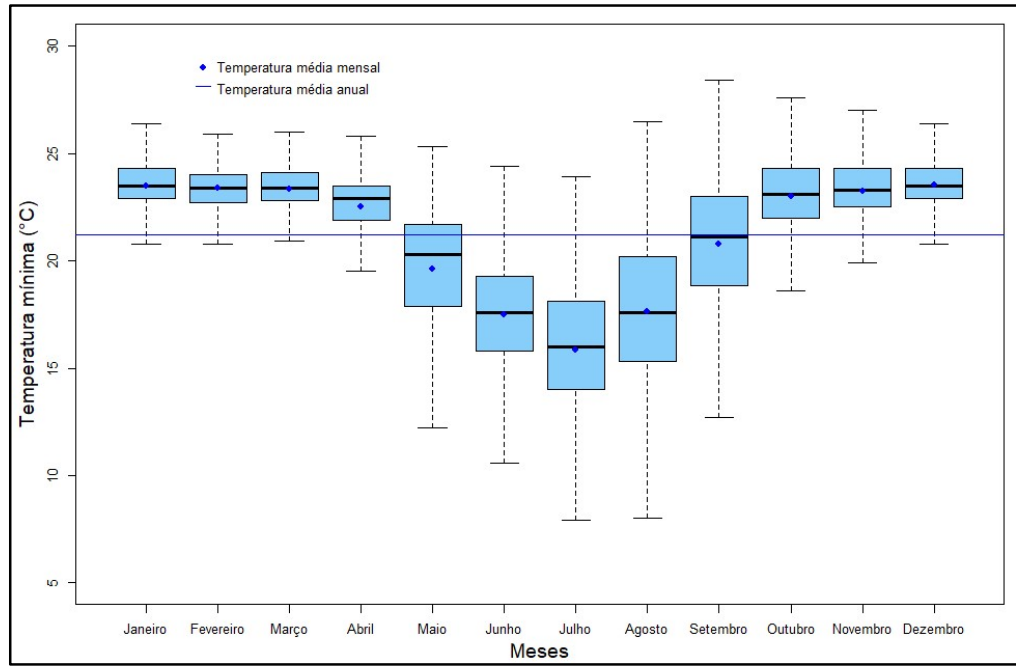

Figura 1: Diagrama de caixa da temperatura mínima de cada mês do ano com as temperaturas médias anual e mensal para o município de Santo Antônio de Leverger-MT. 
Na Tabela 2 apresentam-se as principais estatísticas descritivas mensais para a temperatura máxima diária, obtida na estação meteorológica de Santo Antônio de Leverger-MT. Nessa Tabela, constata-se que as medidas de posição foram superiores ao limite máximo de $32{ }^{\circ} \mathrm{C}$, recomendado para a avicultura ou ficaram próximas a esse limite, como foram os meses de maio, junho e julho. As medidas de dispersão foram maiores para o período compreendido entre os meses de maio e outubro, cujos valores de desvio padrão e coeficiente de variação superaram, respectivamente, $3{ }^{\circ} \mathrm{C}$ e $9 \%$. Com base nesses resultados infere-se que houve uma elevada variabilidade de temperatura na região durante os meses de inverno.

Tabela 2: Estatísticas descritivas mensais para a temperatura máxima diária na Estação Meteorológica Convencional Padre Ricardo Remetter, localizada no município de Santo Antônio de Leverger-MT.

\begin{tabular}{|l|l|l|l|l|l|l|}
\hline \multirow{2}{*}{ Meses } & \multicolumn{5}{|l|}{ Estatística descritiva } \\
\cline { 2 - 7 } & Média & Mediana & Desvio Padrão & Coeficiente de Variação (\%) & Erro Padrão & Intervalo de confiança (95\%) \\
\hline Janeiro & 32,6532 & 32,8000 & 2,2706 & 6,9536 & 0,0717 & $\pm 0,1405$ \\
\hline Fevereiro & 32,4716 & 32,7000 & 2,2354 & 6,8840 & 0,0734 & $\pm 0,1442$ \\
\hline Março & 32,7968 & 33,1000 & 2,0849 & 6,3569 & 0,0652 & $\pm 0,1278$ \\
\hline Abril & 32,6642 & 33,3000 & 2,7455 & 8,4052 & 0,0874 & $\pm 0,1714$ \\
\hline Maio & 31,0758 & 32,4000 & 3,7201 & 11,9712 & 0,1164 & $\pm 0,2282$ \\
\hline Junho & 31,0644 & 32,8000 & 4,1448 & 13,3425 & 0,1344 & $\pm 0,2634$ \\
\hline Julho & 31,5365 & 33,5000 & 4,8398 & 15,3467 & 0,1552 & $\pm 0,3043$ \\
\hline Augusto & 34,1826 & 35,7000 & 4,3545 & 12,7388 & 0,1410 & $\pm 0,2763$ \\
\hline Setembro & 34,9774 & 36,3000 & 4,5544 & 13,0210 & 0,1497 & $\pm 0,2935$ \\
\hline Outubro & 34,9354 & 35,4000 & 3,2247 & 9,2304 & 0,1029 & $\pm 0,2017$ \\
\hline Novembro & 33,6661 & 34,0000 & 2,6949 & 8,0049 & 0,0873 & $\pm 0,1712$ \\
\hline Dezembro & 33,0211 & 33,4000 & 2,3705 & 7,1788 & 0,0752 & $\pm 0,1474$ \\
\hline Total anual & 32,9068 & 33,4000 & 3,6262 & 11,0197 & 0,0335 & $\pm 0,0657$ \\
\hline
\end{tabular}

As estatísticas descritivas anuais do presente estudo, para a temperatura diária máxima do munícipio de Santo Antônio de Leverger-MT, foram semelhantes às obtidas por Salgado et al. (2010) no município de Tupi Paulista-SP. Também, os resultados desta pesquisa concordaram com as estimativas de Araújo et al. (2010) para o município de Iguatu-CE, cujas temperaturas máximas diárias tiveram a sua maior frequência no intervalo entre $32{ }^{\circ} \mathrm{C}$ e $35^{\circ} \mathrm{C}$. Entretanto, Costa et al. (2012) relataram médias de temperaturas máximas diárias próximas a $30,3{ }^{\circ} \mathrm{C}$ para o município de Bananeiras-PB, valor inferior ao limite máximo. De acordo com os resultados deste trabalho, deduz-se que a temperatura no município analisado não foi adequada para o desenvolvimento da avicultura, necessitando-se, portanto, de um custo adicional com a climatização do ambiente nos galpões.

Os diagramas de caixa ('box plot') para a temperatura diária máxima, em função do mês encontramse na Figura 2. Nessa Figura, foram acrescentados os pontos correspondentes à média mensal, e a linha horizontal no valor $32,9068{ }^{\circ} \mathrm{C}$ para o valor médio anual. Nota-se que houve pouca variabilidade dos valores de temperaturas durante os meses de janeiro até abril e de novembro a dezembro. Esses períodos apresentaram menor amplitude térmica, menor diferença entre a média e a mediana, maior proximidade à linha de temperatura média anual e maior tendência de estabilidade térmica. Entretanto, os meses entre maio e outubro evidenciaram elevada amplitude térmica, notável diferença entre a média e a mediana, bem como entre os quartis, inclusive com valores médios de temperatura muito distantes da média anual. Essas características de assimetria podem elevar a probabilidade de ocorrer temperaturas muito diferentes de um dia para outro, agravando-se a situação de conforto ambiental das aves e seu controle, mesmo para os meses de maio a julho, em que as médias foram inferiores a $32{ }^{\circ} \mathrm{C}$. 


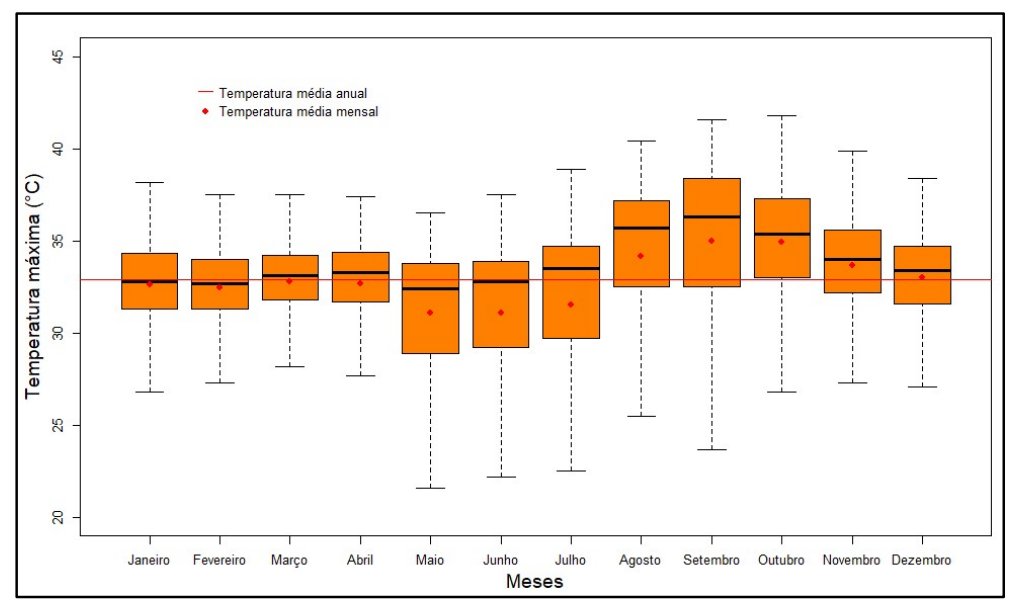

Figura 2: Diagrama de caixa da temperatura máxima de cada mês do ano com as temperaturas médias anual e mensal para o município de Santo Antônio de Leverger-MT.

A aderência da distribuição dos dados de temperatura diária mínima e máxima em cada mês às funções densidade de probabilidade (FDP) foi verificada pelo teste de Qui-Quadrado $\left(X^{2}\right)$, cujos resultados encontram-se na Tabela 3. Nessa Tabela pode-se constatar que os valores da temperatura mínima diária se ajustaram adequadamente à distribuição Normal para todos os meses. Porém, os valores de temperatura máxima diária para os meses de maio até setembro foram modelados aplicando-se a distribuição Gama. Isso concorda com o esperado, pois nas Figuras 1 e 2 foram observadas a simetria dos dados para a temperatura mínima e a assimetria para a temperatura máxima nesses meses.

Tabela 3: Resultados do teste de aderência de Qui-Quadrado $\left(X^{2}\right)$.

\begin{tabular}{|c|c|c|c|c|c|c|}
\hline \multirow{3}{*}{ Meses } & \multicolumn{6}{|l|}{ Temperatura extrema } \\
\hline & \multicolumn{3}{|l|}{ Mínima } & \multicolumn{3}{|l|}{ Máxima } \\
\hline & $\begin{array}{ll}\begin{array}{l}\text { Distribuição } \\
\text { probabilidade }\end{array} & \text { de } \\
\end{array}$ & $\begin{array}{l}\text { Estatística do } \\
\text { teste }\end{array}$ & Probabilidade & $\begin{array}{ll}\text { Distribuição } & \text { de } \\
\text { probabilidade } & \\
\end{array}$ & $\begin{array}{l}\text { Estatística do } \\
\text { teste }\end{array}$ & Probabilidade \\
\hline Janeiro & Normal & 70,0000 & 0,1988 & Normal & 90,0000 & 0,2313 \\
\hline Fevereiro & Normal & 70,0000 & 0,2833 & Normal & 80,0000 & 0,2424 \\
\hline Março & Normal & 90,0000 & 0,2313 & Normal & 80,0000 & 0,2424 \\
\hline Abril & Normal & 50,0000 & 0,2815 & Normal & 70,0000 & 0,2544 \\
\hline Maio & Normal & 80,0000 & 0,2424 & Gama & 90,0000 & 0,2313 \\
\hline Junho & Normal & 70,0000 & 0,1918 & Gama & 80,0000 & 0,2424 \\
\hline Julho & Normal & 80,0000 & 0,2424 & Gama & 90,0000 & 0,2313 \\
\hline Augusto & Normal & 90,0000 & 0,2313 & Gama & 90,0000 & 0,2313 \\
\hline Setembro & Normal & 90,0000 & 0,2314 & Gama & 90,0000 & 0,2313 \\
\hline Outubro & Normal & 50,0000 & 0,2673 & Normal & 50,0000 & 0,2815 \\
\hline Novembro & Normal & 72,5000 & 0,2181 & Normal & 72,5000 & 0,2181 \\
\hline Dezembro & Normal & 90,0000 & 0,2315 & Normal & 90,0000 & 0,2313 \\
\hline
\end{tabular}

A distribuição de probabilidade Normal apresentou a maior aderência aos dados diários de temperatura mínima e máxima no município de Santo Antônio de Leverger-MT, conforme o teste de QuiQuadrado (Tabela 3). O adequado ajuste da distribuição Normal aos dados diários de temperatura mínima indica sua eficiente aplicação em diferentes épocas do ano sem restrições para períodos específicos. Estes resultados corroboram as conclusões formuladas por Assis et al. (2004), Astolpho et al. (2005) e Sansigolo (2008), utilizando a distribuição Normal para estimar a probabilidade de ocorrência de temperaturas mínimas absolutas anuais em diversas localidades do estado de São Paulo. Em relação às temperaturas máximas diárias para os meses de janeiro até abril e entre outubro e dezembro, os resultados do presente estudo 
concordam com os obtidos por Araújo et al. (2010). Esses autores ajustaram, de forma satisfatória, os dados de temperatura máxima diária nesses períodos à distribuição Normal e Gama. Contudo, os referidos autores não obtiveram adequada aderência dos dados no mês de julho à distribuição Gama, como foi constatado nesta pesquisa. Essas discrepâncias podem estar associadas às características do inverno de cada região, pois no Mato Grosso esse período não é estável, podendo ocorrer elevadas temperaturas e flutuações da amplitude térmica diariamente, o que favorece a assimetria da distribuição.

Com base nos modelos de distribuição definidos para cada mês e temperatura extrema (mínima e máxima), determinou-se as probabilidades de ocorrência das categorias de temperaturas classificadas como muito fria, adequada e muito quente para a avicultura, no município de Santo Antônio de Leverger-MT (Figura 3). Nesse gráfico pode observar que na maior parte do ano prevalecem as temperaturas extremas prejudiciais à avicultura, isto é, muito frias ou muito quentes. Nota-se, ainda, que nos meses com maior probabilidade de ocorrer temperaturas adequadas (janeiro, fevereiro, outubro e dezembro), esses valores não ultrapassam um terço dos dias no mês, ou seja, em torno de 10 dias. Apesar da avicultura ser uma atividade promissora no Mato Grosso e inclusive como fonte importante de renta para muitos produtores de agricultura familiar, requer investimentos extras para a climatização das instalações, com a finalidade de melhorar o conforto térmico animal e elevar os indicadores de desempenho produtivo e reprodutivo das aves de corte e postura.

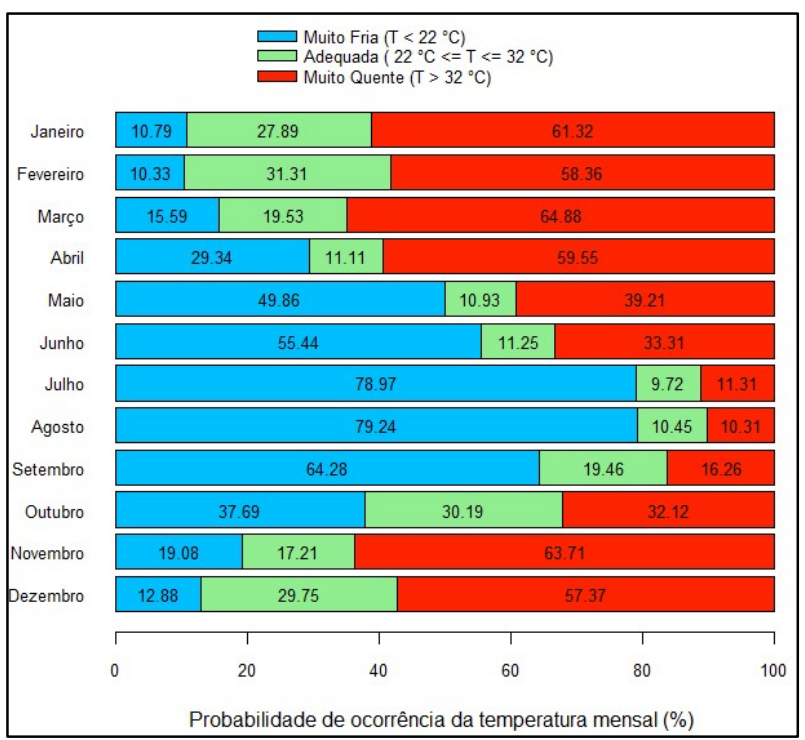

Figura 3: Distribuição das probabilidades de ocorrência da temperatura mensal de cada categoria para o município de Santo Antônio de Leverger-MT.

\section{CONCLUSÕES}

As Funções Densidade de Probabilidade (FDP) foram adequadas, precisas e confiáveis para o ajuste dos dados diários de temperaturas extremas (mínima e máxima) em cada mês do ano, no município de Santo Antônio de Leverger-MT. Os modelos obtidos possibilitaram definir os limites favoráveis para o desenvolvimento da avicultura e estimar a probabilidade de ocorrência das três categorias de temperaturas nesse município. A metodologia adotada apresentou-se como excelente ferramenta para predizer os valores de temperaturas mínimas e máximas ao longo do ano no referido município, auxiliando no planejamento da 
construção de aviários e seu respectivo sistema de ventilação.

AGRADECIMENTOS: Ao Instituto Nacional de Meteorologia (INMET) por possibilitar a consulta dos dados.

\section{REFERÊNCIAS}

ARAÚJO, E. M.; SILVA, I. N.; OLIVEIRA, J. B.. Aplicação de seis distribuições de probabilidade a séries de temperatura máxima em Iguatu-CE. Revista Ciência Agronômica, Fortaleza, v.41, n.1, p.36-45, 2010.

ASSIS, J. P.; DOURADO NETO, D.; MANFRON, P. A.; MARTIN, T. N.; SPAROVEK, G.; TIMM, L. C.. Ajuste de séries históricas de temperatura e radiação solar global diária às funções densidade de probabilidade normal e log-normal, em Piracicaba, SP. Revista Brasileira de Agrometeorologia, Santa Maria, v.12, n.1, p.113-121, 2004.

ASTOLPHO, F.; CAMARGO, M. B. P.; PEDRO JUNIOR, M. J.; PALLONE FILHO, W. J.; BARDIN, L.. Regionalização de riscos de ocorrência de temperaturas mínimas absolutas anuais para o estado de São Paulo com base em modelos probabilísticos e digitais de elevação. Bragantia, Campinas, v.64, n.1, p.139-148, 2005

BORGES, G. M. R.; THEBALDI, M. S.. Estimativa da precipitação máxima diária anual e equação de chuvas intensas para o município de Formiga, MG, Brasil. Revista Ambiente e Água, Taubaté, v.11 n.4, 2016. DOI: https://dx.doi.org/10.4136/ambi-agua.1823

CALDEIRA, T. L.; BESKOW, S.; MELLO, C. R.; FARIA, L. C.; SOUZA, M. R.; GUEDES, H. A. S.. Modelagem probabilística de eventos de precipitação extrema no estado do Rio Grande do Sul. Revista Brasileira de Engenharia Agrícola e Ambiental, Campina Grande, v.19, n.3, p.197-203, 2015. DOI: http://dx.doi.org/10.1590/18071929/agriambi.v19n3p197-203

COELHO, D. J. R.; TINOCO, I. F. F.; VIEIRA, M. F. A.. Mapeamento de aviários de postura abertos em sistema vertical de criação. Revista Brasileira de Engenharia Agrícola e Ambiental, Campina Grande, v.19, n.10, p.996-1004, 2015. DOI: http://dx.doi.org/10.1590/18071929/agriambi.v19n10p996-1004

COSTA, J. H. S.; SARAIVA, E. P.; SANTOS, L. F. D.. Efeito do ambiente sobre indicadores fisiológicos na produção de frangos de corte. Revista Verde de Agroecologia e Desenvolvimento Sustentável, Mossoró, v.7, n.4, p.54-58, 2012.

INMET. Instituto Nacional de Meteorologia. BDMEP: banco de dados meteorológicos para ensino e pesquisa. Brasília: INMET, 2020.
JERSZURKI, D.; SOUZA, J. L. M.; EVANGELISTA, A. W. P.. Distribuição de probabilidade e movimento temporal da precipitação na região de Telêmaco Borba, Paraná. Revista Brasileira de Ciências Agrárias, Recife, v.10, n.1, p.110-116, 2015. DOI: https://doi.org/10.5039/agraria.v10i1a5159

NAVAS, T. O.; OLIVEIRA, H. F.; CARVALHO, F. B.; STRINGHINI, J. H.; CAFÉ, M. B.; HELLMEISTER FILHO, P.. Estresse por calor na produção de frangos de corte. Revista Eletrônica Nutritime, Viçosa, v.13, n.1, p.4550-4557, 2016.

PEEL, M. C.; FINLAYSON, B. L.; MCMAHON, T. A.. Updated world of the Köppen-Geiger climate classification. Hydrology and Earth System Sciences, Göttingen, v.11, n.5, p.16331644. 2007. DOI: https://doi.org/10.5194/hess-11-1633$\underline{2007}$

R CORE TEAM. R: A language and environment for statistical computing. Viena: R Foundation for Statistical Computing, 2020.

SALGADO, D.; NÄÄS, I. A.. Avaliação de risco à produção de frango de corte do estado de São Paulo em função da temperatura ambiente. Revista Engenharia Agrícola, Jaboticabal, v.30, n.3, p.367-376, 2010.

SANSIGOLO, C. A.. Distribuições de extremos de precipitação diária, temperatura máxima e mínima e velocidade do vento em Piracicaba, SP (1917-2006). Revista Brasileira de Meteorologia, São José dos Campos, v.23, n.3, 341-346, 2008. DOI: http://dx.doi.org/10.1590/S010277862008000300009

SILVA, J. B.; LLOPART, M. P.; BOIASKI, N.. Temperatura máxima do ar em Pelotas, RS - Tabelas de probabilidades em escalas de tempo pentadal. Revista Brasileira de Meteorologia, São José dos Campos, v.20, n.2, p.267-276, 2005.

VITORASSO, G.; PEREIRA, D. F.. Análise comparativa do ambiente de aviários de postura com diferentes sistemas de acondicionamento. Revista Brasileira de Engenharia Agrícola e Ambiental, Campina Grande, v.13, p.788-794,

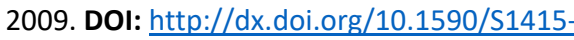
43662009000600018

A CBPC - Companhia Brasileira de Produção Científica (CNPJ: 11.221.422/0001-03) detém os direitos materiais desta publicação. Os direitos referem-se à publicação do trabalho em qualquer parte do mundo, incluindo os direitos às renovaç̃ões, expansões e disseminações da contribuiç̃o, bem como outros direitos subsidiários. Todos os trabalhos publicados eletronicamente poderão posteriormente ser publicados em coletâneas impressas sob coordenação da Sustenere Publishing, da Companhia Brasileira de Produção Científica e seus parceiros autorizados. Os (as) autores (as) preservam os direitos autorais, mas não têm permissão para a publicação da contribuição em outro meio, impresso ou digital, em português ou em tradução. 